\title{
IV. Ueber die Prognose der Herzkrankheiten.
} Von E. Leyden.

(Fortsetzung aus No. 20.)

Die dritte Form, die Herzschwäche, weakened heart, Insufficienz des Herzens, kann ebenfalls aus sehr verschiedenen Ursachen hervorgehen. Die Symptome desselben sind so evident und leicht erkennbar, dass die Diagnose in der Regel augenfällig ist. Die Schwäche der Herzaction documentirt sich durch Kleinheit des Pulses, welcher bald sehr frequent, bald langsam, leicht unterdrückbar ist, allgemeiner Schwäche, auffälliger Blässe und Kälte, Ohnmachten, Schwindel. Beklemmung und Angstgefühle. Dass ein Zustand, in welchem der Herzmuskel zu erlahmen droht, sehr beunruhigend und gefahrvoll ist, leuchtet ein. Die Frage, ob und unter welchen Umständen diese Herzschwäche überstanden werden kann, ist eine sehr dringende. Zunächst kommt die diagnostische Frage in Betracht, ob das Herz organisch erkrankt ist oder nicht. Für die Prognose ist diese Unterscheidung sehr wichtig, obwohl im Augenblick auch die Schwäche eines nicht organisch erkrankten Herzens sehr gefährlich sein kann. Im Allgemeinen aber ist diese functionelle Herzschwäche von geringerer Bedeutung. Bei jugendlichen, neurasthenischen, besonders hysterischen Individuen, gestaltet sich die Prognose allemal günstiger, zumal wenn man bereits weiss, dass Patient an ähnlichen Anfällen gelitten und sie gut überstanden hat. Jugendliches Alter, weibliches Geschlecht, facies composita, lässt die Herzschwäche günstiger beurtheilen, obgleich die Symptome fast immer bedrohlich und beängstigend sind.

Am gefährlichsten ist die acute (plötzlich eintretende) Herzschwäche bei fieberhaften Krankheiten, und zwar sowohl auf der Höhe des Fiebers, wie namentlich zur Zeit des Fieberabfalls und im Beginn der Reconvalescenz. Der mit Recht so gefürchtete Collaps der fieberhaften Krankheiten und die plötzlichen Todesfälle im Beginn der Reconvalescenz beruhen wesentlich auf schnell eintretender Herzschwäche. Fast ebenso gefährlich ist die schnell eintretende Herzschwäche bei Angina pectoris und bei den anämischen Fettherzen, obwohl diese Zustände sehr wohl überwunden werden können. Jedenfalls sind derartige Zufälle so bedeutungsvoll, dass der Arzt die ersten Zeichen derselben rechtzeitig erkennen muss, um der Katastrophe Einhalt thun zu können.

Die chronischen Fälle von Herzschwäche (von Stokes als weakened heart bezeichnet) betreffen alle chronischen Herzerkrankungen, mit und ohne gleichzeitigen Klappenfehler.

Sie entstehen alsdann, wenn der Herzmuskel zu schwach wird, um die ihm zufallende Arbeitsleistung zu absolviren. Infolge desseu resultiren jene Zustände der Stauung, der Herzinsufficienz, welche den Compensationsstörungen bei Klappenfehlern entsprechen und als solche am besten bekannt sind. Entweder ist die Arbeitsleistung: für das Herz abnorm gesteigert, wie bei Klappenfehlern, Arteriosklerose, Nierenerkrankungen, oder bei normalen Widerständen ist die Herzkraft gesunken durch Erkrankungen des Myocard (Myocalditis), oder Ernährungsstörungen desselben (Anämie), oder Intoxication (Alkohol etc.), oder Dilatation (idiopathische Dilatation). Die Prognose dieser chronischen Herzinsufficienz wird einerseits von dem Grade derselben, oder aber wesentlich von der Ursache abhängen. Progressive Erkrankungen, wie Arteriosklerose, Nierenkrankheiten, 
welche zu Herzschwäche führten, geben nur geringe Aussicht auf völlige Wiederherstellung, während Zustände der Intoxication, der mangelhaften Ernährung, wie auch der Ueberanstrengung wohl einer Restitution fähig sind. Die Verhältnisse sind hier sehr analog den Compensationsstörungen bei Herzklappenfehlern, und die Prognose hängt grösstentheils von dem Zustande des Herzmuskels, sowie von der Intensität der Folgezustände $a b$. Wir müssen auf diese Verhältnisse noch weiter unten zurückkommen. Hier sei nur soviel bemerkt, dass neben der medicamentösen Behandlung die neuen Methoden der Herztherapie, namentlich gerade die mechanische Behandlung, weitergehende Erfolge verspricht, als sie bisher elreicht werden konnten. Dieselben sollen im Stande sein, nicht nur die Herzschwäche zu überwinden, sondern sogar eine deutliche Znnahme der Herzmuskulatur zu bewirken. Der unzweideutige Beweis hierfür ist freilich nicht ganz leicht zu führen, und ich glaube, dass wir noch weitere kritisch geprüfte Erfahrungen abwarten müssen, ehe ein abschliessendes Urtheil möglich ist. Dabei scheint es mir von geringerer Bedeutung zu entscheiden, ob der günstige Effect gerade auf die Herzgymnastik zuräckzuführen ist, oder vielmehr auf die allgemeine Besserung der Ernährung, der Kräftigung und Leistungsfähigkeit des ganzen Organismus, an welcher sich auch das Herz in hervorragender Weise betheiligt. Auch von den warmen Bädern wird eine anregende und stärkende Wirkung auf die Herzmuskulatur gerühmt, welche sogar mit derjenigen der Digitalis verglichen wird.

Mit einigen Bemerkungen wollen wir noch des Fettherzens gedenken, welches unter den Herzmuskelerkrankungen besonders genannt zu werden verdient. Wir müssen, wie ich schon früher auseinander gesetzt habe, zwei Formen von Fettherz unterscheiden: a) das Herz der Fettleibigen, b) den fettig degenerirten Herzmuskel.

Das Herz der Fettleibigen bietct verschiedene Formen und Grade dar, welche auch verschieden beurtheilt werden müssen. So lange es sich nur um eine ungewöhnliche Fettanhäufung auf dem Herzen und dem Pericardium handelt, während der Herzmuskel selbst gesund ist, so lange bleibt die Prognose relativ günstig. Selbst dann, wenn sich schon hydropische Erscheinungen darbieten, ist die Prognose nicht schlecht, und man sieht auch in den Hospitälern solche Fälle nicht selten geheilt werden. Eine geeignete diätetische Behandlung, begleitet von methodisch geübter Muskelaction, nach den Principien der Oertel'schen Methode bringt solche Fälle zur Heilung. Wenn aber bereits weitere Veränderungen am Herzen vorhanden sind, insbesondere Dilatationen des linken Ventrikels mit ausgesprochener Herzschwäche, so wird die Prognose sehr unsicher, sie kann für den Moment sehr bedrohlich werden. Noch mehr ist das der Fall, wenn sich bei älteren fettleibigen Personen zu dem einfachen Fettherzen Arteriosklerose hinzugesellt, welche langsam fortschreitet und im allgemeinen unheilbare Zustände hervorruft. Die bedenkliche Dilatation des Fettherzens bezieht sich wesentlich auf den linken Ventrikel. Eine Vergrösserung des Herzens nach rechts hin, auf dem Sternum und darüber hinaus, ist ohne wesentliche prognostische Bedeutung, zumal diese Dämpfung bei Fettleibigen häufig ist und von der reichlichen Fettentwickelung am Pericard herrührt.

Die zweite Form, welche auch häufig als Fettherz bezeichnet wird, ist die fettige Degeneration des Herzmuskels. Ich habe schon vor Jahren darauf hingewiesen, dass diese letztere Form als solche kaum der anatomischen Diagnose unterliegt, dass sie nur mit Wahrscheinlichkeit aus den Symptomen deutlicher Herzschwäche bei schlecht genåhrten und anämischen Individuen zu erschliessen ist. Handelt es sich hier um einfache Anämie oder Schwäche, so ist die Prognose nicht ungünstig, dagegen bei allen schweren Anämieen und Kachexieen geben auch die begleitenden Herzaffectionen ungünstige Prognose, wie überhaupt diese Formen zum grossen Theile von der Bedeutung des Allgemeinzustandes abhängig sind.

Wir kommen nun

ad 3) zur Besprechung der Prognose der Herzklappenfehler.

Hier ist zunächst die wichtige Frage aufzuwerfen, ob Klappenfehler überhaupt völlig heilen können. Diese Frage ist gerade in der deutschen Litteratur mehrfach bearbeitet worden. Eine der ersten und wichtigsten Arbeiten rührt von dem älteren.Jaksch in Prag her (1860), welcher zuerst mit Sicherheit nachwies, dass Herzklappenfehler vollkommen heilen können. Gerhardt hat in seinem Lehrbuch der Auscultation und Percussion 1868 über mehrere geheilte Fälle berichtet (2 Mitralfehler, 1 Aortenfehler), sodann hat Benecke (Zur Therapie des acuten Gelenkrheumatismus und der mit ihm verbundenen Herzkrankheiten), als er auf die Heilkraft der Nauheimer Thermen aufmerksam machte, über 55 Herzfehler berichtet, welche durch den Gebrauch dieser Bäder gebessert, resp. geheilt waren. Auch Herr Geh. Rath G. Meyer in Aachen hat in seiner Schrift: Ueber heilbare Formen chronischer Herzleiden 1881, aus seiner eigenen Praxis einige Fälle geheilter Herzfehler (2 Mitralinsufficienzen) mitgetheilt. Endlich hat Herr Lewinsky in unserem
Verein am 17. April 1882 einen interessanten Vortrag zur Prognose der Herzfehler gehalten, an welchen sich eine lebhafte Discussion anschloss. Herr Lewinsk y zeigte, dass die Mehrzahl der bekannten Fälle geheilter Klappenfehler Mitralinsufficienzen waren, dass aber auch Aorteninsufficienzen heilen können, wofür er einen Fall beibringt, bei welchem s. Z. die Diagnose der Aorteninsufficienz von Traube selbst gestellt war und bei welchem Herr Lewinsky die Heilung constatirte. In der Discussion berichtete Herr Badt über einen Fall von Heilung einer Insufficienz der Aorta, Herr Fräntzel über 2 Fälle von Mitralinsufficienz, 1 von Stenose und ich selbst 2 Fälle von Aorteninsufficienz. Diesen erschöpfendell Darstellungen der deutschen Litteratur ist es kaum nöthig Weiteres hinzuzufügen. Ich beschränke mich daher nur noch auf die Mittheilung einer exquisiten Beobachtung, welche ich in der englischen Litteratur gefunden habe.

In der Discussion, welche sich an den Vortrag von Broadbent anschloss, erwähnt Dr. Campbell Black folgende Beobachtung: Bei einem jungen Menschen von 15-16 Jahren wurden zufällig zwei Aortengeråusche entdeckt, ohne dass er irgend welche $\mathrm{Be}-$ schwerden hat. Nach 5 Jahren verheirathet er sich gegen den Rath der Aerzte, hat Kinder etc. 5 Jahre später wird bei einer erneuten gelegentlichen Untersuchung des Herzens constatirt, dass die Geräusche vollkommen verschwunden sind. Patient ist und bleibt gesund.

Aus allen diesen Beobachtungen geht unzweifelhaft hervor, dass Herzklappenfehler heilen könnell, dass aber die grosse Mehrzahl der Heilungen Mitralinsufficienzen betrifft. Zwar ist es erwiesen, dass auch Aorteninsufficienz heilen kann, aber doch verhältnissmässig sehr selten. Unter Heilung müssen wir natürlich nicht nur das Verschwinden des Geräusches, sondern auch das dauernde Verschwinden aller Herzsymptome verstehen. Der Cardinalpunkt liegt aber in dem dauernden Verschwinden des Geräusches. Dieser Beweis hat nun für die Aorteninsufficienz mancherlei Bedenklichkeit. Denn es ist erwiesen, dass das diastolische Regurgitationsgeräusch an den Aortenklappen verschwinden kann, ohne dass die Insufficienz und deren mechanische Folgen ausgeglichen sind. Bei den experimentellen Untersuchungen über künstlich erzeugte Herzfehler, welche von E. Klebs, O. Rosenbach, Jager, François Frank angestellt wurden, zeigte sich, dass bei Kaninchen trotz der Zerstörung der Aortenklappen nur selten ein diastolisches Geräusch hörbar war. Ferner ist von mir u. a. beobachtet, dass in Fällen, wo sich Aorteninsufficienz mit Aortenstenose combinirt, das diastolische Geräusch verschwindet, sobald die Stenose einen hohen Grad erreicht hat. Endlich hat Herr Stabsarzt Renvers kürzlich in einer Abhandlung: Casuistische Beiträge zur Lehre von der relativen Insufficienz der Aortenklappen, Charité-Annalen 1888, p. 223, gezeigt, dass bei der durch arteriosklerotische Erweiterung der Aorta ascendens bedingten Aorteninsufficienz das Geräusch auftritt, sowie mit erhöhtem Blutdruck eine Dehnung des Aortenrings stattfindet, dagegen in der Ruhe bei gelindem Aortendruck dasselbe Geräusch verschwindet. Hierauf sei ein Theil der als geheilt bezeichneten Fälle von Aorteninsufficienz $\mathrm{zu}$ beziehen.

Es ergiebt sich also die Thatsache, dass Herzfehler, welche unzweifelhaft constatirt sind, im Laufe der Zeit vollkommen geheilt werden konnten, und zwar nicht bloss Mitralfehler, sondern auch Aortenfehler. Bei letzteren indessen ist eine solche wirkliche und dauernde Heilung so selten, dass der Arzt sie für die Prognose nicht wohl in Betracht ziehen darf. Dagegen gestattet die Mitralinsufficienz eine gewisse Hoffnung auf Heilung, und zwar besonders in denjenigen Füllen, wo sie noch frisch ist und sich aus einer acuten Krankheit entwickelt hat. Die vollständige Heilung wird um so eher zu hoffen sein, wenn sich noch keine Folgezustände, abhängig voll der Klappeninsufficienz entwickelt haben.

Wenn nun immerhin die Heilung eines Klappenfehlers zu den Ausnahmefällen zu rechnen ist, so muss in zweiter Linie die Frage aufgeworfen werden, wie ist die Prognose eines Klappenfehlers zu stellen, welcher nicht heilt.

Die Erfahrung hat unzweifelhaft gelehrt, dass Herzklappenfehler, durch unzweidentige physikalische Symptome constatirt, bestehen können, ohne dass der Patient irgend eine Beschwerde davon fühlt. Der Patient wird häufig aus irgend einer anderen Ursache untersucht, und dabei wird der Herzfehler zufällig entdeckt. Diese Thatsache, dass Herzfehler zufällig entdeckt werden, dass der Patient sich dabei vollkommen wohl fühlt, ist bereits seit geraumer Zeit bekannt. Wir bezeichnen dies Verhältniss als das Stadium der vollkommenen Compensation. Die Störungen, welche sich aus den
Klappenerkrankungen für die Mechanik des Blutlaufes ergeben könnten, sind durch erhöhte Leistung der hinter der mechanischen Störung gelegenen Herzabschnitte ausgeglichen. Für die Praxis kommt es natürlich sehr darauf an, welche Prognose bei solchen Patienten, die sich vollkommen wohl befinden, $\mathrm{zu}$ sțellen isț. 
Bis vor nicht langer Zeit wurde ein solcher Patient, obgleich er sich ganz gesund fühlte, doch als ein Todescandidat angesehen, welcher in jedem Augenblick sterben konnte. "Haeret lateri letalis arundo." Der Patient selbst gab sich für verloren, die Eltern des arglosen Kindes sahen mit tiefem Schmerz im Herzen auf ihren zum Tode verurtheilten Liebling. Diesen schweren pessimistischen Anschauungen gegenüber ist es ein Fortschritt, dessen Bedeutung nicht unterschätzt werden darf, wenn wir aus der ärttlicheu Erfahrung mehr und mehr entnommen haben, dass eine so vollkommene Compensation viele Jahre lang bestehen, und dass der Patient während dieser Zeit sich seines Lebens in ungetrübter Weise erfreuen kann. Heutzutage sind solche Erfahrungen keine Seltenheit mehr, und jeder erfahrene Arzt wird ein oder mehrere Beispiele dieser Art aus seiner Praxis im Gedächtnisse haben. Dementsprechend war es ein entschiedenes Verdienst, als Sir Andrew Clark in London in seinem Vortrage, dessen ich schon Eingangs Erwähnung that, sich auf 684 Fälle seìner eigenen Erfahrung berufen konnte, in welchen einc chtonische Klappenkrankheit am Herzen mindestens fünf Jahre lang bestanden hatte, ohne dass ihre Anwesenheit durch Symptome angezeigt war, welche die Gesundheit merklich störten. Der Patient kam wegen anderer Beschwerden (mit Dyspepsie) zum Ar\%te, und der Herzfehler wurde zufällig entdeckt. In vielen Fällen besteht das Stadium der ungetrübten Compensation viel länger, weit über fü̈nf Jahre hinaus bis zu $20 \mathrm{Jahren}$, aber es ist sicherlich bemerkenswerth, dass in einer so beträchtlichen Anzahl von Fällen das jahrelange Fehlen aller Herzsymptome constatirt werden konnte.

Diese Thatsache ist von grosser praktischer Bedeutung. $\mathrm{Zu}$ nächst folgt daraus, dass wir nicht jeden Herzkranken als Todescandidaten ansehen und so behandeln sollen, sondern dass ihm seine Unbefangenheit möglichst bewahrt werde. A. Clark führt Fälle an, wo der Patient seit der Diagnose des Herzfehlers, wie ein Schwerkranker behandelt, von der Welt abgeschlossen eine trübselige Existenz fỉhrte und dadurch, dass er seinem Beruf und den erlaubten Lebensfreuden zurtickgegeben wurde, ermuthigt und aufgerichtet und sich fast als geheilt betrachtete. So richtig dieses ärztliche Verfahren ist, so scheint es mir doch zu weit gegangen, wenn $\mathrm{B}$ r is tow $\mathrm{e}$ sagt: "So lange Jemand mit einer Herzaffection keine Beschwerden hat, soll er auch nichts davon erfahren und seine gewohnte Lebensweise fortsetzen. " Ich gehöre gewiss nicht zu denjenigen, welche den Arzt fthr verpflichtet halten, seinen Patienten oder deren Angehörigen allemal die volle Wahrheit über ihre Zustände zu eröffnen. Im Gegentheil, häufig würde die glückliche Unbefangenheit dem Patienten verloren gehen, sowie er Kenntniss von einem an sich unheilbaren Uebel hat. Dies Wissen ist oft viel schlimmer, als die Krankheit selbst. Aber ein Patient mit einem Herzfehler darf doch nicht so uneingeschränkt leben, wie ein Gesunder und muss soviel über seine Krankheit wissen, dass er sich nicht Schädlichkeiten aussetzt, welche seinen Zustand verschlimmern könnten. O ertel sagt ganz mit Recht: „Es ist ein gefährlicher Irrthum, dass ein Kranker mit gut compensirtem Klappenfehler keiner besonderen Vorschriften bedürfe." Im Gegentheil, er kann seinen relativen Gesundheitszustand nur dadurch dauernd erhalten, dass er gewisse Schädlichkeiten vermeidet, unter denen namentlich die körperliche Ueberanstrengung zu nennen ist, aber auch andere Schädlichkeiten, wie Erkältung, Alkoholgenuss, Tabak, Kaffee u. a. in's Gewicht fallen.

Praktisch kommt ferner noch die Frage in Betracht, welchen Lebensberuf ein Kind mit Herzfehler später erwählen soll, namentlich ob z. B. der Militärdienst möglich ist. Die letztere Frage wird fast ausnahmslos zu verneinen sein, dagegen scheint es mir nicht unbedingt nothwendig, einem Patienten, welcher sich als Soldat einen Herzfehler zuzieht, unbedingt zu rathen, sofort seinen Beruf zu quittiren. Die Mitralinsufficienz bedingt jedenfalls nicht unbedingt diesen Entschluss. - Auch die Lebensversicherung kommt in Betracht. In England scheint es, dass ein gut compensirter Klappenfehler nicht die Annahme zur Lebensversichernng ausschliesst, bei uns dürfteu solche Kranke wohl nicht angenommen werden. Endlich noch die Frage der Verheirathung. Die Ehe ist für Herzkranke nicht ganz unbedenklich. Herzkranke Männer können sich im Geschlechtsgenusse Schädlichkeiten zuziehen, bei Frauen setzt die Schwangerschaft wesentliche Störungen. Sehr leicht, jedoch keineswegs immer, bekommen herzkranke Frauen während der Schwangerschaft Compensationsstörungen mit Hydrops. Ich habe eine Reihe solcher Fälle auf meiner Klinik gesehen. Die Mehrzahl dieser Fälle erholte sich wieder nach der Entbindung, die Entbindung selbst ging gut von statten, die Compensation stellte sich wieder her, aber einzelne hatten irreparable Störungen davongetragen und erholten sich nicht wieder.

Wenn nun also die Erfahrung lehrt, dass Herzklappenfehler in vielen Fällen lange im Stadium vollständiger Compensation verharren können, ohne merkliche Beeinträchtigung der Gesundheit; andererseits aber auch, dass viele Herzfehler sich rapid verschlimmern und zum Tode führen, so müssen wir suchen, im ein- zelıen Falle. die Prognose noch präciser stellen zu können, d. h. zu sagen, ob der vorliegende Fall ein solcher ist, welcher ein langes Stadium der Compensation hoffen lässt, oder welcher vermuthlich einen schlimmen Verlauf nimmt. Für die Beurtheilung dieses Theiles der Prognose ergeben sich folgende Anhaltspunkte:

a) Zunächst kommt in Betracht, wel ch e Klappe erkrankt ist, und in welcher Art (ob Insufficienz oder Stenose). Unter allen Klappen.. krankheiten giebt die Mitral in sufficie $\mathrm{nz}$ die beste Prognose, d. h. sie giebt die beste Garantie sowohl für eine etwaige Heilung, wie für lange Erhaltung der Compensation. Die schlechteste Prognose giebt im Durchschnitt die Insufficienz der Aortenklappen, für welche noch die üble Chance des plötzlichen Todes in Betracht kommt. Nächst dieser ist die Mitralstenose zu nennen. Um jedoch Missverständnisse meiner Angaben zu vermeiden, hebe ich ausdrücklich hervor, dass auch Mitralinsufficienz und Mitralstenose mitunter sehr ungünstig verlaufen, und dass Fälle von Aorteninsufficienz bekannt sind, wo dieser Klappenfehler ohne merkliche Symptome 10-15 und 20 Jahre bestanden hat. Ich selbst kenne seit 15 Jahren einen Herrn mit diesem Klappenfehler, welcher, vollständig compensirt, demselben bis zum heutigen Tage keine Beschwerden bereitet hat.

Die Prognose der Aorteninsufficienz bietet grosse Verschiedenheiten dar. Sie tritt bekanntlich in zwei Formen auf, die eine, endocarditische, giebt cet. par. eine bessere Prognose als die zweite, arteriosklerotische, die sich in höherem Alter (zuweilen ungewöhnlich frïhzeitig) entwickelt, demnach einem progressiven Processe ihren Ursprung verdankt. Zu erwähnen ist noch, dass das Vorhandensein des diastolischen Tones an der Aorta (neben dem diastolischen Geräusch) die Prognose besser gestaltet, indem es auf eine relativ geringfügige Insufficienz schliessen lässt.

b) Die Folgezustände am Herzen kommen ferner in Betracht Je weniger die Hypertrophie (Dilatation) des rückwärts gelegeneu Herzabschnittes ausgeprägt ist, um so günstiger ist die Prognose. Erhebliche Dilatationen sind ungünstiger, am ungünstigsten starke Dilatationen des linken Ventrikels bei Insufficienz der Aortenklappen.

c) Die Intensität der Geräusche.

Man sollte glauben, dass der Herzfehler um so schwerer ist, je lauter die daron abhängigen Geräusche auftreten. Dies ist aber, wie namentlich Englische Autoren angeben, nicht der Fall. In Gegentheil, laute distincte Geräusche sind günstiger als die verschwindenden. Es ist bekannt, dass bei Mitralstenosen, wenn sie sehr intensiv werden, das präsystolische Geräusch mehr oder weniger vollständig verschwindet, analog ist es bei Aortenfehlern der Fall. ${ }^{1}$ ) Icl kann diese Erfahrungen aus meiner Beobachtung vollkommen bestätigen.

d) Die Natur des endocarditischen Processes, insbesondere bezüglich der Frage, ob derselbe ein stationärer oder ein progressiver ist. Die Wichtigkeit dieser Frage leuchtet ein. Es giebt eine Anzahl von Klappenerkrankungen, welche man als stationäre bezeichnen darf, in einer anderen Reihe von Fällen hat der Process ausgesprochene Neigung zum progressiven Fortschritt.

Vollkommen stationär sind in der Regel die angeborenen Herzfehler, der Process ist abgelaufen, recidivirt nicht, und die gut etablirte Compensation wird ohne besondere Zwischenfälle nicht gestört. Ich kenne eine junge Dame, die seit 12 Jahren zu meinel Patientinnen gehört und mich damals wegen eines Herzfehlers aufsuchte, welcher von einigen Aerzten für ein Aneurysma erklärt wurde. Ich habe eine angeljorene Insufficienz der Pulmonalklappe constatirt. Patientin hatte keine weiteren Beschwerden, als dass sie zuweilen die Geräusche fühlte, zuweilen auch nur zu fühlen glaubte. Der $\mathrm{Zu}$ stand ist bis hente vollkommen derselke. Patientin hat sich an ihren Herzfehler gewöhnt und hat gelernt, ihn nicht weiter zu beachten. Uebrigens ist Patientin Zwilling und hat sich ebenso kräftig und schnell entwickelt, wie ihre Schwester, welche keinen angeborenen Herzfehler besitzt. Fast ebenso günstig sind die Verhältnisse bei Klappenfehlern nach acuten Krankheiten, und unter diesen am besten bei Mitralinsufficienzen nach Chorea. Man weiss, dass sich bei Chorea nicht selten Herzklappenfehler entwickeln, am häufigsten Mitralinsufficienz, selten Aortenfehler. Die ersteren sind nicht nur stationär, sondern heilen ziemlich häufig, wogegen die Aortenfehler stationär bleiben. (A. Clark stellt den Satz auf: die Geräusche der Mitralinsufficienz nach Chorea verschwinden meist in 8-9 Jahren nach der Attaque.)

Unter den fieberhaften acuten Krankheiten führt, wie bekannt,

1) Broadbent sagt: "Die physikalischen Zeichen tăuschen häufig. Kein grösserer Fehler, als die Gefahr eines Klappenfehlers nach der Stärke des Gerăusches abzumessen" - und an einer anderen Stelle: "Ein lautes Geräusch ist in der Regel weniger bedenklich, als ein schwaches und sanftes. Denn dieses bedeutet Herzschwäche, welche die grösste von allen Gefahren bedingt." - Auch Gairdner sagt: "Halbgeübte Untersucher neigen dazu, die Bedeutung der Geräusche zu überschätzen und dem blossen Verhalten der Töne zuviel Aufmerksamkeit zuzuwenden, während sie die übrigen Umstände unterschätzen, unter denen das Geräusch auftritt.“ 
der Gelenkrheumatismus nicht selten zu Endocarditis und Klappenfehlern. Seltener, aber unzweifelhaft auch, Masern, Scharlach, Diphtherie, Typluss. Der endocarditische Process dieser Fälle ist ein benigner und disponirt nicht zu progressivem Fortschreiten, der Gelenkrheumatismus bietet die Gefahr des Recidivs dieser Krankheit, mit welchem häufig eine Exacerbation der Endocarditis verbunden ist. Dagegen sind die Herzfehler nach Masern und Scharlach, welche die Patientell fast ausnahmslos nur einmal befallen, stationär und geben eine relativ gute Prognose. Dabei versteht es sich von selbst, dass auch hier cet. par. die Mitralinsufficienz der günstigste, die Aorteninsufficienz der ungünstigste Klappenfehler ist.

$\mathrm{Zu}$ den progressiven und daher ungünstigen Formen der Klappenfehler gehörer alle diejenigen, welche durch Arteriosklerose bedingt sind, auch die durch Ueberanstrengung entstandenen sind häufig progressiv. Indessen muss doch auch daran erinnert werden, dass viele Fälle von Aortenfehlern bei älteren Individuen, welche ohne Zweifel zur Arteriosklerose gehổren, Jahre lang stationär bleiben können.

Endlich ist noch die Form der fieberhaften oder septischen Endocarditis zu erwähnen. Dass diese Form eine verhältnissmässig bösartige ist, liegt auf der Hand. Sie ist häufig, aber nicht immer, verbunden mit ausgeprägten Klappenerkrankungen, namentlich an den Aortenklappen, und entwickelt sich, obne dass Gelenkrheumatismus besteht oder vorangegangen ist. Das Fieber ist ein continuirliches oder remittirendes oder intermittirendes, ausgezeichnet durch chronisches Fortbestehen trotz aller dagegen angewandten Mittel. Die Consumption durch das andauernde Fieber bedingt eine üble Prognose, zuweilen kommt noch Embolie im Gehirn, den Extremitäten etc. hinzu. So übel die Prognose ist, so giebt es doch auch - wie ich schon früher berichten konnte - Fälle, welche nach längerem Fieberverlaufe in Genesung übergehen. Ich sah erst kürzlich einen solchen Fall, wo das Fieber nahezu 7 Monate andauerte, und wo neben rheumatischen Gelenkaffectionen eine Insufficienz derAortenklappen bestand. Schliesslich hat das Fieber nach und nach aufgehört, der Patient befindet sich in einem leidlichen Zustande.

Selbstverständlich kommen für die Prognose der Herzklappenfehler auch die allgemeinen Verhältnisse in Betracht, deren ich schon oben gedachte. Alter, Geschlecht, Constitution und Ernährung, Charakter, Intelligenz sind ebenfalls für die Prognose in mehr oder minder ausgedehntem Maasse nicht ohne Bedeutung, kurz eine grosse Mannichfaltigkeit der Verhältnisse, die nicht allein die Prognose beeinflussen, sondern gleichzeitig die Gefahren bezeichnen, welche der Herzkranke läuft, und damit Mittel und Wege an die Hand geben, um solche Gefahren und Störungen zu vermeiden, dagegen die günstigen Einflüsse aufzusuchen und wirksam zu machen.

(Fortsetzung folgt.) 